\title{
A new hand gestures recognition system
}

\author{
Ahmed Kadem Hamed AlSaedi ${ }^{1}$, Abbas H. Hassin AlAsadi ${ }^{2}$ \\ ${ }^{1}$ Faculty of Information Technology, University of Babylon, Iraq \\ ${ }^{1}$ Faculty of Education, University of Misan, Iraq \\ ${ }^{2}$ Faculty of Information Technology, University of Basrah, Iraq
}

\begin{tabular}{l} 
Article Info \\
\hline Article history: \\
Received Jul 26, 2019 \\
Revised Sep 24, 2019 \\
Accepted Oct 8, 2019 \\
\hline Keywords: \\
Computer vision \\
Gestures recognition \\
Hand gestures \\
OpenCV library \\
PyAutoGUI library
\end{tabular}

Corresponding Author:

Abbas H. Hassin,

College of Information Technology,

Basrah University, Iraq.

Email: abbas.hassin@uobasrah.edu.iq

\begin{abstract}
Talking about gestures makes us return to the historical beginning of human communication, because, in fact, there is no language completely free of gestures. People cannot communicate without gestures. Any action or movement without gestures is free of real feelings and cannot express the thoughts. The purpose from any hand gestures recognition system is to recognizes the hand gesture and used it to transfer a certain meaning or for computer control or and device. Our paper introduced a low cost system to recognize the hand gesture in real-time. Generally, the system divided into five steps, one to image acquisition, second to pre-processing the image, third for detection and segmentation of hand region, four to features extraction and five to count the numbers of fingers and gestures recognition. The system has coded by Python language, PyAutoGUI library, OS Module of Python and the OpenCV library.
\end{abstract}

Copyright $@ 2020$ Institute of Advanced Engineering and Science. All rights reserved.

\section{INTRODUCTION}

Historically, the Electronic Visualization Lab was the first to create a data glove called Sayre Glove this was 1977 [1]. Thirty-five years later, the researchers adopted the camera to interact with the computer. In fact, the camera compared to the data glove is considered more direct and natural to achieve Human Computer Interaction [2].

Recently, the interactive by gesture has become widely used and in the future may replace the mouse and/or keyboard by vision-based devices. The main feature of using hand gestures is to interact with the computer as an input unit [3]. The gesture is defined as a form of nonverbal communication or non- vocal communication where the body's movement can convey certain messages. Gestures originate from different parts of the human body, but the most common ones emerge from the hand or face.

Gesture provides a new form for interaction that reflects the experience of user in the actual world. The interaction by gesture be more natural and do not require any hindering or additional hardware. There are two kinds of gestures, static gestures and dynamic gestures. In [4] Liang introduced the best definition for hand posture or static gesture and dynamic hand gesture as: instance."

"Posture is a specific combination of hand position, orientation, and flexion observed at some time

"Gesture is a sequence of postures connected by motion over a short time span."

The good examples of static hand gesture are "OK" or "STOP" and "No", "Yes", "goodbye" for dynamic gestures.

Three approaches were used to obtain important information for any hand gesture recognition system, Data gloves approaches, Vision based approaches, and Color-Marker approaches as shown strictly in Figure 1. 
In Vision based approaches [5, 6], the human motion obtained from one camera or more [7, 8], and the devices based on vision can handle many properties for interpreting the gesture, for example, color and texture whereas the sensor has not this property. Although these approaches are simple, many challenges can appear for example lighting diversity, complex background, and presence objects with skin-color similar to the hand (clutter), as well as the system, requires some criteria like time of recognition, speed, durability, and efficiency of computation [9-11].

Data glove approaches use types of sensors to capture the position and motion for the hand. In these approaches can compute in easy and accurate the coordinates of the locations of the fingers palm, and hand configurations [10-16]. The sensors do not achieve an easy connection with the computer because it needs to be the user connected physically with the computer and hinder the movement of the hand. These devices are also expensive, and unsuitable to operate in an environment of virtual reality [13]. According to Moore's Law, the sensors will become smaller and cheaper over time. It will prevalent in the future, we believe.

Colored- Markers approaches used marked gloves worn by the hand of a human and be colored to help in the process of the hand tracking and to locate the fingers and palm. Marker gloves can form the shape of hand by extract the geometric-features [17]. In [18] used a wool glove with three different colors to represent the palms and fingers. This approach considers simple and not expensive if compared with Sensor or Data Glove [18], but the natural interaction between human and computer still not enough [17, 19].

However, there are some challenges when we want to design a strong and real-time gesture recognition system. Challenges related to complex hand structures that lead to difficulty in tracking and recognition. In addition, there are challenges related to the shape of the gesture, the different lighting conditions, the real time issue and the presence of noise in the background. These challenges taken into account in this paper by used running average principle in Background subtraction technique to detect and extract the hand from background and used contour of hand as feature.

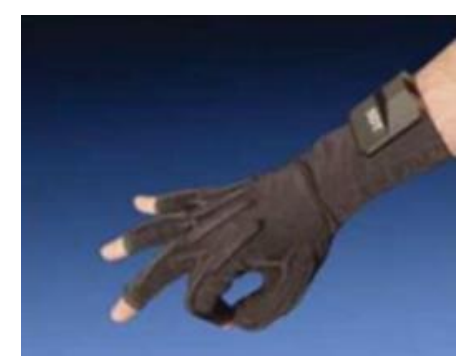

(a) "Data-Glove approaches" [12]

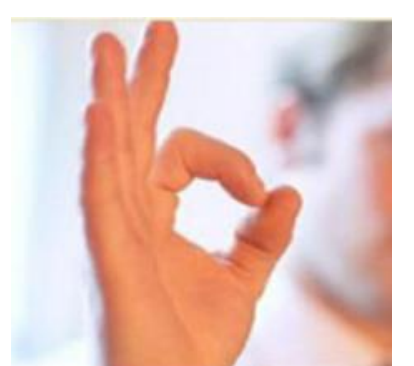

(b) "Vision-Based approaches "

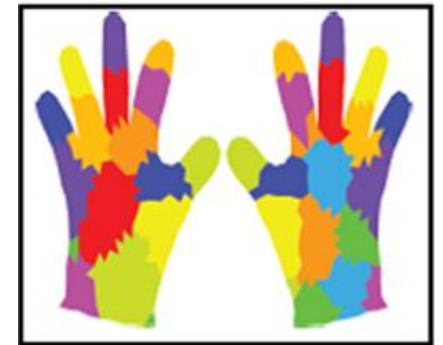

(c)"Colored Marker approaches"

Figure 1. Examples of input techniques for hand gesture recognition system

There are many systems designed to recognize the hand gesture, we will mention to some of them. In [20] Amiraj and Vipul introduced a system to recognize the hand gestures for HCI. They used more than one approach for preprocessing step in their system and used two methods to perform segmentation process one with a static background and another without the constraint of background. In static background used constant value to a threshold, they used Otsu thresholding algorithm, and when the thresholding is dynamic used the color at the real-time mode. In the free background constraint, used thresholding methods based on color feature and subtraction of background model. For detecting the hand, they found the contour of it and then computing the convex hull and convexity defect to find the number of fingers. They provided three approaches to interact with devices, tracking of the finger, orientation of the hand, and counting of fingers.

Shwetha k., Yewale et al. [21] provided a review for many hand gesture recognition systems that used Matlab language. They used a Canny edge algorithm to determine the edge of hand and used the values of hue and saturation for Skin -Color detection. They concluded that the system gave better results when used Artificial Neural Networks (ANNs) and edge detection methods.

In [22], Nancy et al, introduced a system to hand gestures recognition by using Color-Marker approach. The user wearing white cloth in your hand and place a red color marker on hand's fingertip. The gestures in this system used to point on the computer's screen by detection the only finger with a red color marker. However, this system does not achieve direct contact with the devices because of it used the color marker.

In [23] introduced a hand gestures recognition system by Tasnuva Ahmed. The system based on neural networks and in real time. The researcher divided the system into four steps, image capturing, 
pre-processing, features extraction, and recognition. The system succeeded in distinguishing hand gestures that taking from different angles or size or orientations but there is a delay in the system due to the training phase of the Artificial Neural Network as well as delay in switching between the nodes. Badgujar et.al presented a dynamic hand gestures classification system using contour analysis. It is an efficient system for computer control, but it applies only to PowerPoint presentations [24].

\section{RESEARCH METHOD}

The proposed system was coded by Python language, PyAutoGUI library for executing operations of mouse or keyboard, OS Module of Python and the OpenCV library. The general structure for any system to recognize the hand gestures can be explained as shown in Figure 2. Our system contains five steps, which are detailed.

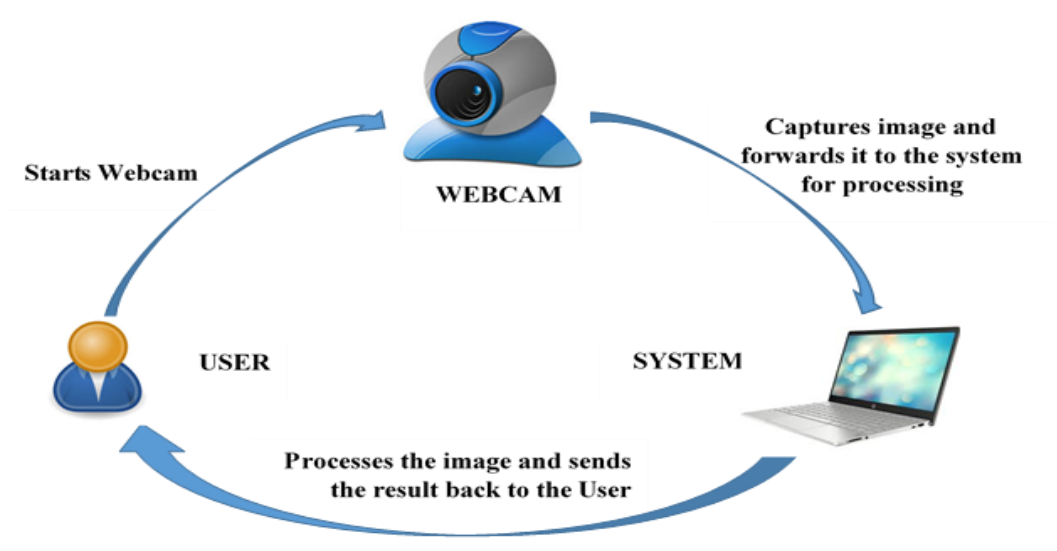

Figure 2. General structure of the hand gestures recognition system

\subsection{Image Capturing}

In this step used a webcam to acquire the RGB image (frame by frame) and based on only bare hand without glove or colored marker that can be hinder the user.

\subsection{Pre-Processing}

In this step in order to minimize the computation time we took only the important area instead of the whole frame from the video stream and this is called Region Of Interest (ROI). In image processing prefers to convert the color images into a grayscale images to increase the processing and after complete the processing can restore the images to its original color space, therefore, we convert region of interest into a grayscale image. Then blurring the (ROI) by Gaussian blur to reduce the objects that have high frequency but not the target. Notice that in this step the algorithm will fail if there is any vibration for the camera.

\subsection{Hand Region Segmentation}

This step is important in any system to hand gestures recognition and help in enhancing the performance of the system by removing the unwanted data in the video stream. In general, there are two methods to detect the hand in image, the first method depends on Skin-Color, this method is simple but effected by the light conditions in the environment and the nature of background. The second method does not depend on Skin-Color but on shape of hand and benefit from the principle of convexity in detection of the hand. The shape of hand is very important feature in the systems of recognition the hand gesture [25].

There are several methods or techniques used to extract the hand region from the image can be summarized as:

a) Edge-Detection.

b) RGB values because of the values of RGB for hand different from the background of the image.

c) Subtraction of background

In this paper used background subtraction technique to separate the hand from the background. This technique needs to determine the background that can be obtained by benefit from a running average principle. The background is computing from made the system focus on a certain scene for at least 30 frames and during that calculating the running average for the current frame and all previous frames using the following equation: 


$$
\operatorname{dst}(x, y)=(1-\text { alpha }) \cdot d s t(x, y)+\operatorname{alpha} \cdot \operatorname{src}(x, y)
$$

where, $\operatorname{src}(\mathrm{x}, \mathrm{y})$ is a source image may be one or three channels and 8-bits or 32-bits floating point, dst (x,y) is destination image contains the same channels in the source image and 32-bits or 64-bits floating point. Finally, alpha is a weight of the source image and can be considered as threshold to determine the time for computing the running average over the frames.

After determining the background, we put the hand front the camera, then compute the absolute difference between the background that calculates by using the running average and the current frame that contains the hand as a foreground object. Thus find the background and then compute the difference all that called subtraction background.

The next step is thresholds the image that output from background subtraction and the result will be only hand with white color and the rest image with black color. The threshold process is important and must be done before the contours finding a process to achieve high accuracy. Mathematically can represent the threshold principle as follows:

$$
f(x)=\left\{\begin{array}{c}
1, \text { if } x \geq \text { threshold } \\
0, \text { if } x<\text { threshold }
\end{array}\right.
$$

where, $f(x)$ is the intensity of the pixel.

All the processes above called Motion-Detection. Figure 3 shown the output of Hand region segmentation process. Finally, perform a chain of morphological processes such as erosions and dilations to remove any small regions of noise.

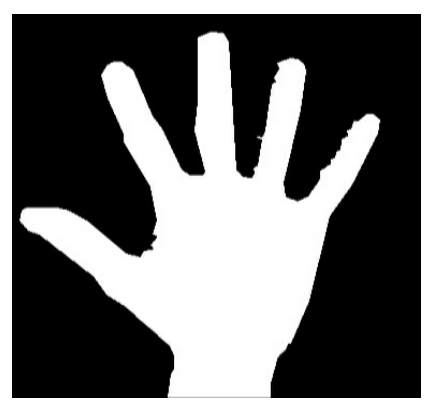

Figure 3. Output of hand region segmentation process

\subsection{Contour-Extraction}

The contour can be defined as object's boundary or outline (hand in our case) that be located in the image. In other words, the contour is a curve connecting points that have the like color value and is a very important in shape analysis, objects detection and recognition process.

\subsection{Features Extraction and Recognition}

Now we turn to the second part of the research, which is how we determine the number of fingers. From the number of fingers can recognize the hand gestures, and for performing this task used Convex Hull to locate the extreme points (top, bottom, left and right). The convex hull is the group of points that surrounding the region of hand as shown in Figure 4. Here, we must clarify the principle of the Convex Set, which means all lines between any two points within hull are entirely within it. From extreme points can compute the palm's center, Figure 5 shown that.

The next step is to draw a circle about the fingers, its center point is the center of the palm and the seventy percent from the length of maximum Euclidean distance between the palm's center and extreme points represent the radius. After that using the bitwise AND operation between the circle from the previous step and the threshold image, the slices of fingers that result from this operation can use to compute the number of hand fingers. After determined the gesture based on the hand's fingers number, the corresponding operation is performed. In fact, the process of distinguishing the hand gesture is a dynamic process. After performing the required instruction from the gesture, return to the first step to take another image to be processed and so on. 


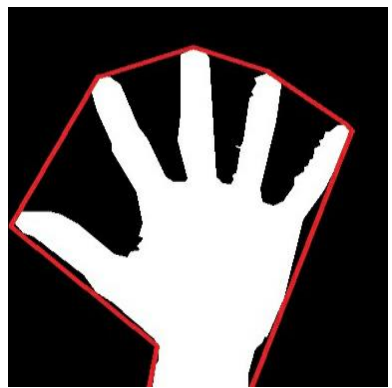

Figure 4. Convex hull

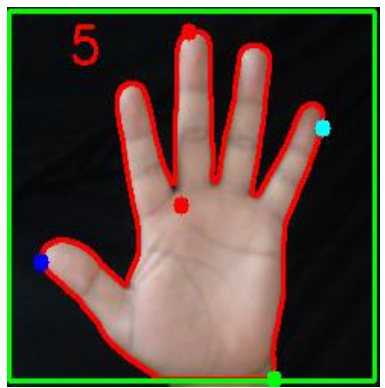

Figure 5. Extreme points (top, bottom, left, right) and center of palm

\section{RESULTS AND DISCUSSION}

This paper recognized six gestures as shown in Figure 6. After recognition step can generate the command that associated with each gesture and passed to control the computer. The six gestures those recognized in this paper used to generate the actions of mouse and used to open various applications such as Microsoft Office (Word, Excel, PowerPoint), VLC Media Player, Google Chrome, Internet Explorer etc. The system implemented with different rotations, scale, lighting and plain or simple background. Table 1 shows the experimental results of proposed system with threshold values $(50,75)$ and alpha value 0.5. The performance of system is given in Chart 1 . The results of system shown that the rate of recognition is $96.6 \%$. The system was applied in different conditions, it has given the best results when the background is clear and the light is medium, the rate of recognition reached $100 \%$.
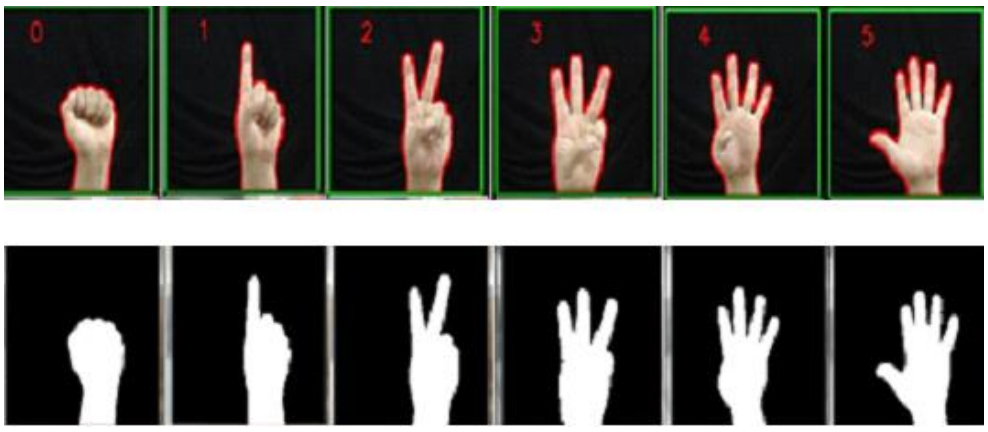

Figure 6. Shape of six gestures

Table 1. Hand Gesture Recognition Rate

\begin{tabular}{ccccc}
\hline Gesture name & No.of input samples & No.of recognized samples & Recognition rate $(\%)$ & Total recognition rate $(\%)$ \\
\hline Zero & 10 & 9 & 90 & \\
One & 10 & 10 & 100 & \\
Two & 10 & 10 & 100 & $\mathbf{9 6 . 6}$ \\
Three & 10 & 10 & 100 & \\
Four & 10 & 10 & 100 & \\
Five & 10 & 9 & 90 & \\
\hline
\end{tabular}

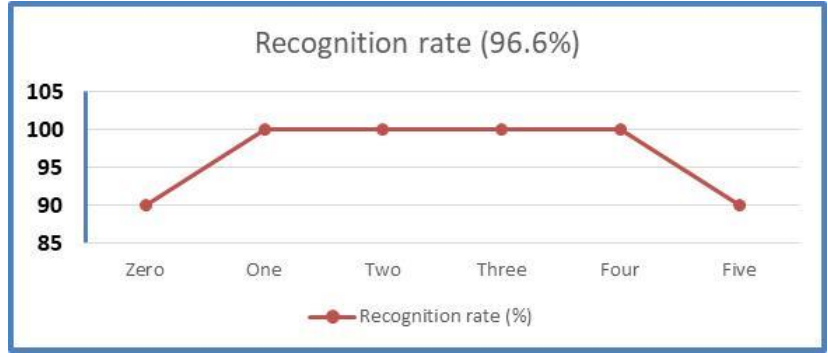

Chart 1. Performance results 


\section{CONCLUSION}

For a long time, the problem of distinguishing gestures was important in computer vision because of the challenge of extraction the target object such as hand from a background that has been cluttered and all that in real-time. In fact, the human when looking to a certain image can easy detecting what is inside it but that very difficult for the computer if looks to the same image because it deals with the image as a matrix with three dimensions.

Our paper solved the challenge of rotation, orientation, scaling, and got the same results when used right or left hand. The system that provided used only bare hand and webcam of Laptop so it is very flexible for the user. The system does not need a database, but it is directly distinguishes the gesture and this achieves the speed of the system. The results of system shown that the rate of recognition is $96.6 \%$ and this result is considered very good compared with other research papers. In future and to enhance the system can use the both hands instead of use only right hand and that will increase the number of gestures. The results section showed that the best rate of recognition is when the background is clear and the light is medium, so these limits must be addressed in the future in order to increase the accuracy of the system.

\section{REFERENCES}

[1] D. J. Sturman and D. Zeltzer, "A survey of glove-based input," in IEEE Computer Graphics and Applications, vol. 14, no. 1, pp. 30-39, Jan. 1994.

[2] B. Ma, W. Xu, and S. Wang, "A Robot Control System Based on Gesture Recognition Using Kinect," TELKOMNIKA (Indonesian Journal of Electrical Engineering), vol. 11, no. 5, pp. 2605-2611, 2013.

[3] N. I. A.M, N. A. Zakaria, S. R and N. M.S, "Analysis of surface electromyography for hand gesture classification," Indonesian Journal of Electrical Engineering and Computer Science (IJEECS), vol. 15, no. 3, pp. 1366-1373, September 2019.

[4] M. V. Lamar, M. S. Bhuiyan, and A. Iwata, "T-CombNET-A Neural Network Dedicated to Hand Gesture Recognition," Biologically Motivated Computer Vision Lecture Notes in Computer Science, vol. 1811, pp. 613-622, May 2000.

[5] L. Chen, F. Wang, H. Deng, and K. Ji, “A Survey on Hand Gesture Recognition,” 2013 International Conference on Computer Sciences and Applications, pp. 313-316, Dec. 2013.

[6] T. B. Moeslund, E. Granum, "A survey of computer vision-based human motion capture", Computer Vision and Image Understanding, vol. 81, no. 3, pp. 231-268, Mar 2001.

[7] S. Bilal, R. Akmeliawati, M. J. E. Salami, and A. A. Shafie, "Vision-based hand posture detection and recognition for Sign Language-A study," 2011 4th International Conference on Mechatronics (ICOM), pp. 1-6, May 2011.

[8] W. N. Khotimah, T. Anggita and N. Suciati, "Indonesian sign language recognition using kinect and dynamic time warping," Indonesian Journal of Electrical Engineering and Computer Science (IJEECS), vol. 15, no. 1, pp. 495-503, July 2019.

[9] G. R. S. Murthy and R. S. Jadon "A review of vision based hand gestures recognition," International Journal of Information Technology and Knowledge Management, vol.2, no. 2, pp.405-410,2009.

[10] P. GARG, N. AGGARWAL and S. SOFAT, "Vision based hand gesture recognition," World Academy of Science, Engineering and Technology, vol. 49, no. 1, pp. 972-977, 2009.

[11] A. Erol, G. Bebis, M. Nicolescu, R. D. Boyle, and X. Twombly, "Vision-based hand pose estimation: A review," Computer Vision and Image Understanding, vol. 108, pp. 52-73, 2007.

[12] L. Dipietro, A. Sabatini, and P. Dario, "A Survey of Glove-Based Systems and Their Applications," IEEE Transactions on Systems, Man, and Cybernetics, Part C (Applications and Reviews), vol. 38, no. 4, pp. 461-482, 2008.

[13] J. Laviola, "A survey of hand posture and gesture recognition techniques and technology," Master Thesis, Brown University, Providence, RI,29, 1999.

[14] R. Samad, L. W. Yan, M. Mustafa, N. R. H. Abdullah and D. Pebrianti, "Multiple Human Body Postures Detection using Kinect," Indonesian Journal of Electrical Engineering and Computer Science (IJEECS), vol. 10, no. 2, pp. 528-536, May 2018.

[15] M. I. Sadek, M. N. Mikhael, and H. A. Mansour, "A new approach for designing a smart glove for Arabic Sign Language Recognition system based on the statistical analysis of the Sign Language", 2017 34th National Radio Science Conference (NRSC), pp. 380-388, 2017.

[16] C. H. Chuan, E. Regina, C. Guardino, "American Sign Language Recognition Using Leap Motion Sensor", 2014 13th International Conference on Machine Learning and Applications, pp. 541-544, 2014.

[17] M. M. Hasan and P. K. Mishra, "Hand gesture modeling and recognition using geometric features: a review," Canadian journal on image processing and computer vision 3.1, pp. 12-26, 2012.

[18] L. Lamberti and F. Camastra, "Real-Time Hand Gesture Recognition Using a Color Glove," Image Analysis and Processing-ICIAP 2011 Lecture Notes in Computer Science, pp. 365-373, Sep. 2011.

[19] R. Wang and J. Popovi, "Real-Time Hand-Tracking with a Color Glove", Proc. ACM Siggraph, vol. 28, no.3, August 2009.

[20] A. Dhawan and V. Honrao, "Implementation of hand detection based techniques for human computer interaction," International Journal of Computer Applications, vol. 72, no. 17, pp. 0975-8887, June 2013. 
[21] S. K. Yewale and P. K. . Bharne, "Hand gesture recognition using different algorithms based on artificial neural network," In: 2011 International conference on emerging trends in networks and computer communications (ETNCC). IEEE, pp. 287-292, 2011.

[22] N. and. G. s. sekhon, "An Analysis of Hand Gesture Recognition Technique using Finger Movement Detection Based on Color Marker," International Journal of Computer Science \& Communication, vol. 3, no. 1, pp. 129-133, January-June 2012.

[23] T. AHMED, " A neural network based real time hand gesture recognition system," International journal of computer applications (0975 - 8887)، vol. 59, no. 4, December 2012.

[24] S. D. Badgujar, G. Talukdar, O. Gondhalekar and S. Y. Kulkarni, "Hand Gesture Recognition System," International Journal of Scientific and Research Publications, vol. 4, no. 2, pp. 2250-3153, Feb. 2014.

[25] L. Li and L. Zhang, "Corner Detection of Hand Gesture," TELKOMNIKA (Indonesian Journal of Electrical Engineering), vol. 10, no. 8, pp. 2088-2094, December 2012.

\section{BIOGRAPHIES OF AUTHORS}

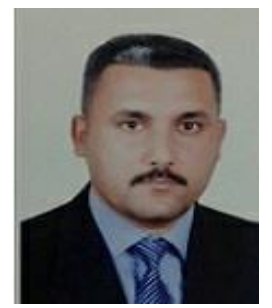

Ahmed Kadem Hamed AL-Saedi obtained his B.Sc. degree from Baghdad University in Computer Science. Has a Diploma in Data Security from Iraq Commission for Computers and Informatics, Institute for Post Graduate Studies in Informatics, Baghdad, Iraq. His M.Sc. (Computer Science) from Basrah University. He is currently a Ph.D. student at Babylon University. His research interests include Computer Vision, Human-Computer Interaction, Medical Image processing and image processing.

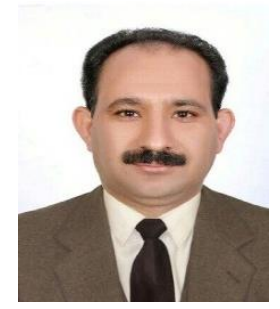

Abbas H. Hassin Alasadi is a Professor at the Information Technology College at Basra University. He received his PhD degree from School of Engineering and Computer Science / Harbin Institute of Technology, China. He spent more than ten years as Associate Professor at different Universities abroad the current position. His research interests include Medical Image processing, Biometrics, Information retrieval, and Human-computer interaction. His research work has been published in various international journals and conferences. Dr.Abbas is an active reviewer in many journals of the areas of computer science and software engineering. He is one of ACIT, UJCS, SIVP, IJIMAI, and IJPRAI members.) 